\title{
Harmonic Skeleton Guided Evaluation of Stenoses in Human Coronary Arteries
}

\author{
Yan Yang ${ }^{1}$, Lei Zhu ${ }^{1}$, Steven Haker ${ }^{2}$, \\ Allen R. Tannenbaum ${ }^{1}$, and Don P. Giddens ${ }^{1}$ \\ 1 Department of Biomedical Engineering, \\ Georgia Institute of Technology, Atlanta, GA, 30332, USA \\ gtg929i@mail.gatech.edu, zlzl@ece.gatech.edu, tannenba@ece.gatech.edu, \\ don.giddens@coe.gatech.edu \\ 2 Surgical Planning Lab, Brigham and Women's Hospital, \\ Harvard Medical School, Boston, MA 02115, USA \\ haker@bwh.harvard.edu
}

\begin{abstract}
This paper presents a novel approach that three-dimensionally visualizes and evaluates stenoses in human coronary arteries by using harmonic skeletons. A harmonic skeleton is the center line of a multi-branched tubular surface extracted based on a harmonic function, which is the solution of the Laplace equation. This skeletonization method guarantees smoothness and connectivity and provides a fast and straightforward way to calculate local cross-sectional areas of the arteries, and thus provides the possibility to localize and evaluate coronary artery stenosis, which is a commonly seen pathology in coronary artery disease.
\end{abstract}

\section{Introduction}

Stenosis is a commonly seen pathology resulting from atherosclerosis, which is a systemic disease of the vessel wall that occurs in the aorta, carotid, coronary, and peripheral arteries [1. Atherosclerotic plaques that develop in vessel walls can intrude into the vessel lumen and result in a narrowing (stenosis) of the lumen. When stenosis occurs in coronary arteries, it will cause insufficient supply of blood to the heart tissue, and sometimes leads to serious results such as heart attacks. The detection of stenoses in vivo can greatly assist the diagnoses and treatments of coronary heart diseases. The conventional way of identifying stenoses is by using coronary angiography imaging, but this method is invasive, and it only provides 2D projections of the vessel lumen, which may yield biased or inaccurate evaluation of the stenosis. Thanks to the development of multisliceCT (MS-CT), we are now able to acquire 3D volumetric data of the entire coronary artery tree, and obtain the complete information of its geometry so that we can perform more accurate measurements based on these data.

Numerous methods have been proposed to extract skeletons of tubular structures in the human body such as blood vessels, airways and colons. Most methods in literature fall into the following two classes [4: boundary peeling (also called thinning, erosion, etc.) [5], and distance coding (distance transform) [2, 3]. Although both classes of methods have yielded promising results, these methods 
usually have problems of preserving connectivity and being smooth. Also, direct extension of these methods to 3D is usually difficult and may not guarantee a unique solution for a single structure.

In this paper, we explore a skeletonization method based on the solution of the Laplace equation with boundary conditions of the first kind (Dirichlet problem) on the surface of a tubular structure. Since the solution of the Laplace equation is a harmonic function, the skeleton extracted using this method is called the harmonic skeleton [8]. The harmonic skeleton is easy to compute, is guaranteed to be smooth, and can automatically give a viewing vector when used as a guidance for fly-throughs for virtual endoscopy. Moreover the space of harmonic functions is linear (as opposed to that of the distance transform on which the ordinary skeleton is founded). The distance transform is commonly used in endoscopy; see $[9$ and the references therein.

Further, we develop an effective way to determine the boundary conditions for solving the appropriate Laplace equation, so that the points that form the final skeleton are evenly distributed, without being too dense or too sparse, thus guaranteeing the connectivity of the skeleton. Most importantly for our applications, the cross-sectional area at a certain location on the artery can be conveniently measured using the least squares plane that fits all the points on the iso-value contour of the solution of the Laplace equation. This means that we can acquire the cross-sectional areas throughout the artery in order to identify stenoses.

We tested our method on CT data sets of both normal and diseased coronary arteries and developed different ways to visualize and analyze stenoses of the arteries. The method is described in detail in Section 2, and we show the results in Section 3.

\section{Methodology}

\subsection{Harmonic Skeleton}

The harmonic skeleton can be obtained by solving the Laplace equation on the surface of the artery. Let $\Sigma$ denote the surface, which is topologically a branched tube. Each branch has a boundary $\sigma_{i}(i=0, \ldots, N-1)$, which is a closed contour, as shown in Figure 1. Here $N$ denotes the total number of boundaries including the root $\sigma_{0}$, which can be selected arbitrarily or selected based on physiological considerations. We have chosen the root $\sigma_{0}$ to be at the proximal section of the left main (LM) coronary artery. Note that we are working on a triangulated representation of $\Sigma$, which makes it convenient for performing numerical computations. The surface can be obtained by segmenting the MSCT images. In this case, we have used a segmentation approach that combines Bayesian classification and active contours [6, 7] to get the required surface for the skeletonization algorithm.

Next we can calculate the harmonic function $u$ by solving the Laplace equation on $\Sigma \backslash \sigma_{i}(i=0, \ldots, N-1)$ :

$$
\Delta u=0
$$




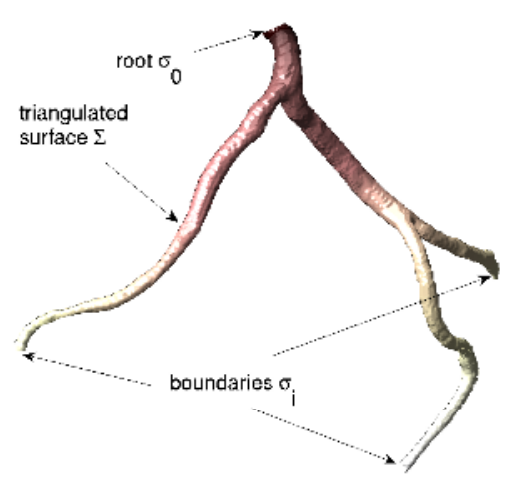

Fig. 1. The segmented and openended surface of left coronary arteries with boundary contours at each end; the surface is colorpainted with solved harmonic function $u$, where dark color means lower $u$, and light color means higher $u$

with proper boundary conditions. To find these boundary conditions, we assign the values at the boundaries in the following manner:

1. Set the boundary value of $u$ at the root $\sigma_{0}$ to be zero, and set StepNumber $=0$;

2. Label all the points on $\sigma_{0}$ as "visited";

3. Label all the neighbors of the "visited" points as "visited";

4. StepNumber ++ ;

5. Repeat steps 3. and 4. until "visited" points reached another boundary $\sigma_{i}$;

6. Set the value at the current boundary $\sigma_{i}$ to be the current value of StepNumber.

In other words, we set the boundary value of $\sigma_{i}$ to be the number of triangle strips between $\sigma_{i}$ and the root boundary $\sigma_{0}$. In coronary arteries, there are several branches, some of which are long and some of which are short. Note that if we used standard Dirichlet boundary conditions in which $u\left(\sigma_{0}\right)=0$ and $u\left(\sigma_{i}\right)=1$ for all $i$ 's, as for example, is done in [13], the resulting points that form the skeleton will be denser for the short branches, and sparser for the longer ones. Hence we employ the boundary conditions stated above to get more evenly distributed skeleton points.

Having obtained the boundary conditions for the Dirichlet problem, we can solve the Laplace equation $\Delta u=0$ on the surface using finite element techniques [6, 12. From the standard theory [10], $u$ exists and is unique, with the maximum and minimum values on the boundaries. In Figure 1, the surface of the artery is painted with the solved $u$, where brighter color indicates higher $u$, and darker color indicates lower $u$.

Using the harmonic function $u$, we can now build the harmonic skeleton. We first find the level sets of $u$ on the surface, i.e., sets $\{x \mid u(x)=\nu\}$ for values of $\nu$ ranging from 0 to $\max (u)$. Each level set corresponds to a group of points on the surface that have the value of a certain $\nu$. These points are not necessarily located on the vertices of our original triangulation of the surface, so we obtain these level points by linearly interpolating the coordinates of the vertices according to the values of $u$. 
We also check the change in the topology of the level contours as we proceed, and further partition these contours into sub-contours if not all the points are connected to form a single contour. This means there is a bifurcation and one contour splits into two at the current level of $u$. We assume one contour cannot split into more than two contours, which is usually true for coronary arteries. The centroid of each contour or sub-contour is then calculated: $\frac{1}{M} \sum_{i=1}^{M} \mathbf{x}_{i}$, where $\mathbf{x}_{i}=\left(x_{i}, y_{i}, z_{i}\right)^{\prime}$ is a point on the contour, and $M$ is the total number of points on the current contour. Each centroid contributes a point to the final harmonic skeleton. By increasing $\nu$ from 0 to the maximum value of $u$, we can find a series of centroids that can finally be connected to build a structured tree, which is the harmonic skeleton.

For the sake of accuracy, an additional step can be performed to refine the harmonic skeleton by replacing the previous boundary conditions with new values obtained as the distance along the skeleton from the root boundary to the other boundaries. We then solve the Dirichlet problem with the new boundary conditions and further obtain a refined harmonic skeleton.

\subsection{Cross-Sectional Area Measurement}

The harmonic skeleton obtained using the above method can be used as a guide in calculating cross-sectional areas at specific locations along the vessels, and the cross-sectional areas can in turn be used to precisely evaluate stenoses in the coronaries. Cross-sectional areas are better than diameters because atherosclerotic plaques are usually eccentric and asymmetric, and the cross-sections of the narrowed vessel lumen may not be perfectly circular. The stenosis may be underestimated if one uses only diameters for stenosis evaluation. Cross-sectional areas, on the other hand, provide more information about the stenosis, and are directly related to the flow rate of blood through the cross-section.

One straightforward way of obtaining the cross-sections of the artery is by looking for a plane that is perpendicular to the skeleton to cut the tube at a certain location. But this strongly depends on the local properties of the skeleton, and a plane that is perpendicular to the skeleton locally may not be perpendicular to the vessel itself. What we do here is to start with the contours or sub-contours obtained in Section 2.1, and measure the cross-sectional area at the same time as we calculate the centroid.

The first thing to do is to find the cross-sectional plane. We have many more points (about 50 points, somewhat more for wider contours and somewhat less for narrower contours) on a level contour than what is necessary (3 points) for determining a plane. Hence we need to use least squares to solve this overdetermined problem. Suppose we have a set of points $X=\left(\mathbf{x}_{1}, \mathbf{x}_{2}, \ldots \mathbf{x}_{N}\right)^{\prime}$, where $\mathbf{x}_{i}=\left(x_{i}, y_{i}, z_{i}\right)^{\prime}, i=1, \ldots, M$ is a point on the level contour, and we build a matrix $A=X-\mathbf{x}_{0}$, where $\mathbf{x}_{0}\left(x_{0}, y_{0}, z_{0}\right)^{\prime}$ is the centroid of all the points on the contour. Calculating the Singular Value Decomposition (SVD) 14 of matrix $A$, we get $A=U S V^{T}$, where $U$ and $V$ are orthogonal matrices and $S$ is a diagonal matrix with non-negative diagonal entries in decreasing order. The singular vector in $V$ that corresponds to the smallest singular value in $S$ is the 
cosine of the direction of the normal to the best-fit plane. The centroid and the normal vector can now be used to uniquely determine the least squares plane.

We now use this plane to cut the surface of the vessel and get a series of points on the surface that also belong to the least squares plane. Again, these points may not coincide with the vertices of the triangulated surface, so we find the points on the triangle edges, and connect them to form a polygon. We then calculate the area of the polygon, and the result is the required cross-sectional area at the current location on the vessel.

\section{Results}

\subsection{Clinical Data}

We tested our method on a healthy volunteer without any clinical symptoms and a patient with plaques in left anterior descending (LAD) coronary artery. The healthy volunteer was imaged using a GE LightSpeed16 CT scanner with a slice spacing of $0.625 \mathrm{~mm}$ and an in-plane resolution of $0.60 \mathrm{~mm}$, while the patient with LAD plaques was imaged using a Siemens Sensation64 CT scanner with a slice spacing of $0.75 \mathrm{~mm}$ and a in-plane resolution of $0.39 \mathrm{~mm}$. Contrast agents were used in both cases. The coronary arteries were extracted by image segmentation using the techniques of [6, 7], and the triangulated surfaces were generated using the Visualization Toolkit (VTK) [15]. We then performed our skeletonization and cross-sectional area measurement methods on the triangulated surfaces.

\subsection{Generation of Harmonic Skeletons}

The harmonic skeletons of both cases were generated using the method described in Section 2.1. Figure2 (Left) and (Middle) show the surfaces in half-transparent mode with the skeletons inside the surfaces, and Figure 2 (Right) shows the skeleton of the diseased (Middle) coronary arteries. The skeletons provide the
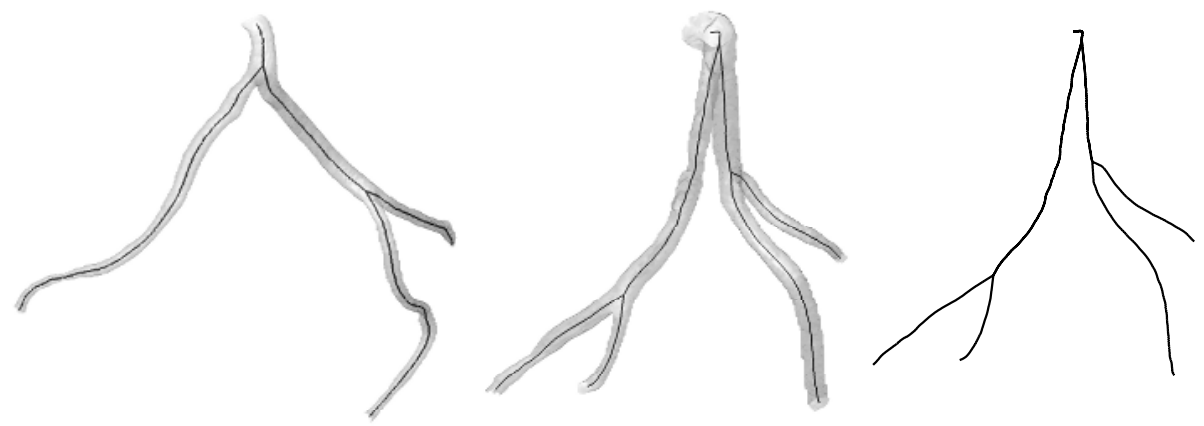

Fig. 2. Left: Coronaries and the skeleton of a healthy volunteer. Middle: Coronaries and the skeleton of a patient with plaques in LAD. Right: The skeleton of the middle coronaries. 
basic information of the arteries, such as bifurcation sites, bifurcation angles and curvatures along the vessels, but they themselves alone are not able to provide much information about the stenosis.

At the bifurcation sites, some post-processing was performed to keep the skeleton smooth and natural. Increasing the value of $u$ and tracking the topology change of the level contours, one contour will split into two at a certain $u$ level, and there will be a jump between the centroid of the single parent contour and the centroids of the two daughter contours. If we connect the centroids directly, the resulting skeleton may not be smooth where it bifurcates. To solve this problem, we start from the daughter branches, and trace back until they reach the bifurcation site, and then extend the branches further until they meet the parent branch. After the post-processing, the skeleton is visually more natural at the bifurcation sites.

\subsection{Stenosis Evaluation}

We calculated cross-sectional areas along the vessel with a resolution of $\delta u=0.5$, which means we measure the cross-sectional area once we increase the harmonic function $u$ by 0.5 . For each coronary artery, there are roughly 400 levels of crosssectional areas, and at each level, there are one or several cross-sectional areas measured according to the number of branches at this level.

We can visualize the quantitative measurements in two different ways. The first way is to specify a $u$ value, and show the cross-sectional areas at this $u$ level, as illustrated in Figure 3 (Left). This is equivalent to showing the areas by specifying a location on the vessel. The second way is to create a color map of the crosssectional area values, and paint the surface with the color of these values. This provides an intuitive way to localize the stenosis, by showing different colors where the cross-sectional area has an apparent change along the vessels, as indicated in Figure 3(Right). In this figure, lighter color indicates normal sized vessel lumen, and darker color means the lumen is narrowed, thus implying stenosis.
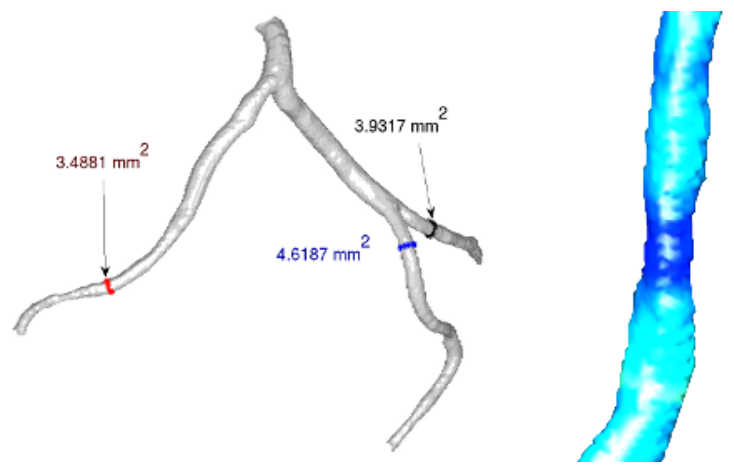

Fig. 3. Cross-sectional area visualization. Left: The cross-sectional area of each branch of the coronaries for a certain $u$. Right: Color-painted LAD indicates a stenosis site in the vessel. 


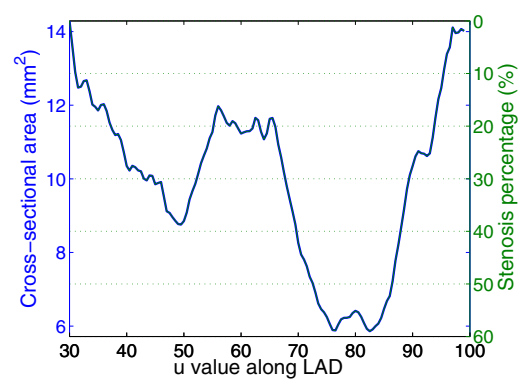

Fig. 4. Cross-sectional area along the LAD shown in Fig. 3 (Right). The left y-axis shows the cross-sectional area in $\mathrm{mm}^{2}$, and the right $y$-axis shows the stenosis percentage.

Figure 4 is a plot of $u$ versus cross-sectional area along the LAD of Figure 3 (Right). The left y-axis shows the cross-sectional areas in $\mathrm{mm}^{2}$, and the right $\mathrm{y}$-axis shows the stenosis percentage, which is an important measure for the evaluation of stenosis. We can see from this plot that this LAD has two stenosis sites, one mild (a little less than $40 \%$ blockage) and another more severe $(60 \%$ blockage). This is in consistent with what is shown in Figure 3 (Right).

\section{Discussion and Conclusions}

In this note, we have proposed an approach to extract harmonic skeletons of tubular structures and applied it to both healthy and diseased coronary arteries. The skeleton generated can be used as a guide for cross-sectional area measurements in order to localize and evaluate stenosis of the arteries. The harmonic skeleton is smooth and unique, and can also serve as a guide in other applications such as virtual endoscopy.

Our algorithm is reasonably fast, taking 2-3 minutes in Matlab to generate the harmonic skeleton from a surface consisting of about 30,000 triangles and 12,000 vertices, on a $1 \mathrm{G} \mathrm{Hz}$ personal computer running Linux. If the skeleton extraction and cross-sectional area measurement are performed at the same time, it takes 6-7 minutes for the same sized data. This makes it possible to use this algorithm in a real clinical setting.

We should also point out that this skeletonization and cross-sectional area measuring approach is performed on a segmented surface of a tubular structure, so the measurement results highly rely on the correctness of the image segmentation. On the other hand, the cross-sectional area can also serve a measure for the validation of segmentation to compare the values of a ground truth model (such as a phantom or a vessel cast) and the segmentation result.

\section{Acknowledgements}

This work is part of the National Alliance for Medical Image Computing (NAMIC), funded by the National Institutes of Health through the NIH Roadmap for Medical Research, Grant U54 EB005149. This work was also supported by a grant from NIH (NAC P41 RR-13218 through Brigham and Women's Hospital). S. Haker's 
work is supported by NIH grants R01CA109246, R01LM007861, R01CA1029246 and P41RR019703.

\section{References}

1. V. Fuster, Z.A. Fayad, J. J. Badimon, "Acute coronary syndromes: biology", Lancet 1999; 353 (supp1 2): SII5-SII9

2. M. Maddah, H. Soltanian-Zadeh, and A. Afzali-Kusha, "Snake modeling and distance transform approach to vascular centerline extraction and quantification", Computerized Medical Imaging and Graphics, 27, 2003, pp. 503-512

3. M. Wan, Z. Liang, Q. Ke, L. Hong, I. Bitter, and A. Kaufman, "Automatic Centerline Extraction for Virtual Colonoscopy", IEEE Trans. on Med. Imag. 21, 2002, pp. $1451-1460$

4. Y. Zhou, and A. W. Toga "Efficient Skeletonization of Volumetric Objects", IEEE Tran. on Visualization and Computer Graphics, Vol. 5, NO. 3, 1999, pp. 196-209

5. L. Hong, A. Kaufman, Y. Wei, A. Viswambharan, M. Wax and Z. Liang, "3D Virtual Colonoscopy", Proc. of IEEE Biomedical Visualization, 1995, pp. 26-32

6. S. Haker, G. Sapiro, A. Tannenbaum, "Knowledge-Based Segmentation of SAR Data with learned Priors", IEEE Tran. on Image Processing, Vol. 9, No. 2, Feb. 2000, pp. 299-301

7. Y. Yang, A. Tannenbaum, and D. Giddens. "Knowledge-Based 3D Segmentation and Reconstruction of Coronary Arteries Using CT Images", In Proceedings of the 26th Annual International Conference of the IEEE EMBS, pages 1664-1666, 2004

8. L. Zhu, S. Haker, and A. Tannenbaum. "Flattening Maps for the Visualization of Multi-branched Vessels", IEEE Trans. on Med. Imag., 24(2):191 - 198, 2005

9. S. Bouix, K. Siddiqi, A. Tannenbaum, "Flux Driven Automatic Centerline Extraction," Medical Image Analysis 9, 2005, pp. 209-221.

10. Stanley J. Farlow "Partial Differential Equations for Scientists and Engineers", Dover Publications, Inc. New York, 1993

11. S. Haker, S. Angenent, A. Tannenbaum, and R.Kikinis, "Nondistorting flattening maps and the 3D visualization of colon CT images", IEEE Trans. Med. Imag., Vol. 19, 2000, pp. 665-670

12. T. Hughes, "The Finite Element Method", Upper Saddle River, NJ: Prentice-Hall, 1987

13. A. J. Yezzi, Jr., J. L. Prince, "An Eulerian PDE Approach for Computing Tissure Thickness", IEEE Trans. Med. Imag., Vol. 22, No. 10, 2003, pp. 1332-1339

14. W. Press, S. Teukolsky, W. Vetterling, and B. Flannery, Numerical Recipes in C, 2nd ed. Cambridge: Cambridge Univ. Press, 1992

15. Kitware Inc. http://www.vtk.org 